\title{
Prevalência de sarcopenia e fatores associados em pacientes submetidos a hemodiálise em um ambulatório em Caxias do Sul/RS
}

\author{
Prevalence of sarcopenia and associated factors in patients submitted to hemodialysis in an ambula- \\ tory in Caxias do Sul/RS
}

DOI: $10.37111 /$ braspenj.2020354013

Nicole Gobbi ${ }^{1}$

Joana Zanotti²

\section{Unitermos:}

Insuficiência Renal Crônica. Sarcopenia. Diálise Renal.

\section{Keywords:}

Renal Insufficiency, Chronic. Sarcopenia. Renal Dialysis.

\section{Endereço para correspondência:} Nicole Gobbi

Centro Universitário da Serra Gaúcha - FSG

Rua Os Dezoito do Forte, 2366 - São Pelegrino - Caxias do Sul, RS, Brasil - CEP 95020-472

Email: gobbinicole@gmail.com

Submissão:

21 de março de 2020

Aceito para publicação

26 de novembro de 2020

\section{RESUMO}

Introdução: Os pacientes portadores de doença renal crônica (DRC) submetidos a hemodiálise (HD), normalmente, apresentam baixo peso associado ao déficit de massa muscular e baixo desempenho físico, sendo frequente o diagnóstico de sarcopenia. Dentre os principais fatores que levam a tal desfecho, destaca-se o hipercatabolismo da doença e a síndrome urêmica. A presença de sarcopenia está associada com as elevadas taxas de mortalidade. $O$ presente estudo tem por objetivo avaliar a prevalência de sarcopenia e fatores associados em pacientes com DRC em hemodiálise em um ambulatório na cidade de Caxias do Sul/RS. Método: Foram coletados dados antropométricos, como peso e estatura, idade para posterior cálculo e classificação do índice de massa corporal (IMC), circunferência da panturrilha $(C P)$, força de preensão manual e teste de caminhada para diagnóstico da sarcopenia. A sarcopenia foi definida conforme critério pré-estabelecido pela European Working Group on Sarcopenia in Older People (EWGSOP). Considerou-se um nível de significância de $5 \%(p \leq 0,05)$ para identificar associação entre as variáveis e o desfecho. Resultados: Dos 95 pacientes avaliados, $21 \%(n=20)$ apresentavam sarcopenia e $36,8 \%(n=35)$ dos sujeitos estavam em situação de risco para o desenvolvimento da mesma. Referente à caracterização da amostra, $61,1 \%(n=20)$ dos pacientes eram do sexo masculino, 53,7\% ( $n=51)$ tinham $<60$ anos. A média de idade encontrada foi de $55,4 \pm 15,1$ anos. A média do IMC foi $25,9 \pm 5,1 \mathrm{~kg} / \mathrm{m}^{2}$, sendo que $45,3 \%(\mathrm{n}=43)$ apresentavam excesso de peso, $34,7 \%(n=3)$ tinham CP indicando depleção muscular. Conclusão: As condições de provável sarcopenia e sarcopenia estiveram presentes na maioria dos pacientes estudados, especialmente nos doentes mais velhos e com baixo peso.

\section{ABSTRACT}

Introduction: The patients with chronic kidney disease (CKD) undergoing hemodialysis in most of the cases present low weight associated with muscle mass deficit and low physical performance, also had been diagnosed with sarcopenia. Within the main factors that lead to such outcome, we highlight the hypercatabolism of the disease and the uremic syndrome. The presence of sarcopenia can lead to associated with high mortality rates. The present study aims to evaluate the sarcopenia prevalence and relate factors of hemodialysis on former of clinic patients at Caxias do Sul/RS city. Methods: Anthropometric data collected, such as weight height and age for subsequent calculation and classification of body mass index (BMI), calf circumference (CC), handgrip strength and walking test for sarcopenia diagnosis. Sarcopenia is defined according to criteria pre-established by the European Working Group on Sarcopenia in Older People (EWGSOP). Results: 95 patients evaluated, $21 \%(n=20)$ had sarcopenia, and $36.8 \%(n=35)$ of the subjects were at risk for its development. Regarding the sample characterization, $61.1 \%(n=20)$ were male, $53.7 \%(n=51)$ were $<60$ years old. The average age found was $55.4 \pm 15.1$ years. The mean BMI was $25.9 \pm$ $5.1 \mathrm{~kg} / \mathrm{m}^{2}$, and $45.3 \%(\mathrm{n}=43)$ were overweight, $34.7 \%(\mathrm{n}=3)$ showed CC indicating muscle depletion. Conclusion: The conditions of probable sarcopenia and sarcopenia were present in most patients studied, especially in older and underweight patients. 


\section{INTRODUÇ̃̃O}

Uma das grandes preocupações para a saúde pública mundial é o aumento da prevalência de pessoas portadoras de doença renal crônica (DRC)'. Conforme o censo da Sociedade Brasileira de Nefrologia (SBN), aproximadamente 133 mil pacientes encontravam-se em tratamento dialítico no Brasil, em 2018, número que vem aumentando desde 2009, sendo neste período aproximadamente 77 mil pacientes ${ }^{2}$. Os pacientes submetidos ao tratamento de hemodiálise (HD) estão sujeitos a complicações agudas e/ou crônicas ${ }^{3}$.

Sendo a DRC uma doença associada com a ocorrência de depleção calórico-proteica, principalmente devido à baixa ingesta alimentar e perda de sangue e nutrientes através da HD, o estado nutricional de pacientes submetidos a HD comumente é comprometido ${ }^{4}$. Além disso, os pacientes podem sofrer com os efeitos da uremia, que se caracteriza pelo excesso de ureia e outros restos metabólicos no sangue, provocando sintomas como náuseas, vômitos e inapetência ${ }^{5}$. Estudos indicam que a prevalência de desnutrição de pacientes em HD gira em torno de $40 \%$, situação cujos desfechos costumam ser desfavoráveis devido ao aumento do risco de morbimortalidade 6 .

A perda de massa magra associada à perda de funcionalidade vem sendo estudada em pacientes portadores de DRC, condição denominada sarcopenia, comum no processo de envelhecimento. Pacientes portadores de DRC são propensos ao desenvolvimento de sarcopenia devido à redução de atividade física, anorexia, acidose metabólica, perda de nutrientes pela hemodiálise, acúmulo de toxinas urêmicas, deficiência de vitamina $\mathrm{D}$ e utilização de múltiplos fármacos, como hipoglicemiantes orais e anti-hipertensivos ${ }^{7,8}$. A presença de sarcopenia em doentes renais crônicos está potencialmente associada a desfechos desfavoráveis, visto que o aumento da prevalência dessa síndrome está relacionado com a piora de função renal e aumento do risco de mortalidade. A prevalência média estimada de sarcopenia em pacientes hemodialíticos, segundo estudos recentes, varia entre 13\% e 33\%. As disfunções metabólicas, carências nutricionais e o aumento do desgaste de massa magra, bem como a dificuldade de regeneração da mesma, justificam a sarcopenia em $\mathrm{HD}^{7,8}$.

Baseado neste contexto, este trabalho tem por objetivo avaliar a prevalência de sarcopenia e fatores associados em pacientes em hemodiálise de um ambulatório de Caxias do Sul/RS.

\section{MÉTODO}

\section{População e Desenho do Estudo}

Trata-se de um estudo observacional com delineamento transversal, realizado no ambulatório de nefrologia de um hospital do município de Caxias do Sul/RS. Os dados foram coletados entre fevereiro e março de 2019. A amostra foi obtida por conveniência. Foram incluídos pacientes com idade $\geq 19$ anos, de ambos os sexos, com DRC em tratamento de hemodiálise, aptos para realizarem avaliação antropométrica (peso, estatura e circunferência da panturrilha), bem como os testes para o diagnóstico da sarcopenia (velocidade de marcha e força de preensão palmar). Foram excluídos pacientes com membros amputados, com dificuldade para locomoção, cadeirantes e acamados. Os dados demográficos, como sexo, idade e tempo de diálise, foram obtidos dos prontuários dos pacientes.

\section{Aferições Antropométricas}

A aferição do peso foi realizada pós diálise, com balança digital para posterior cálculo do IMC (peso/estatura²), com classificação de acordo com a OMS9, para adultos, e Lipschitz $^{10}$, para idosos.

Para avaliação de reserva de massa muscular, a circunferência da panturrilha (CP) foi aferida com fita métrica não elástica, mensurada no perímetro de maior circunferência da panturrilha, ao final da sessão de HD. $\bigcirc$ ponto de corte para homens é $\leq 34,0 \mathrm{~cm}$ e para mulheres $\leq 33,0 \mathrm{~cm}$, como indicativos de baixa reserva muscular ${ }^{11}$.

\section{Aferição da Força de Preensão Manual}

Para avaliar a força de preensão manual (FPM), foi utilizado o dinamômetro manual da marca SAEHAN®, com os pacientes confortavelmente sentados, com o ombro levemente aduzido, cotovelo, quadril e joelho fletido a $90^{\circ}$ e antebraço em posição neutra. A posição do punho pode variar de 0 a $30^{\circ}$ de extensão ${ }^{12}$. Os testes foram realizados no braço sem a fístula, e a força foi aplicada durante 5 segundos por cada tentativa, foram realizadas 3 repetições, entre cada repetição houve um intervalo de 30 segundos. A FPM fora realizada durante a hora inicial da sessão de diálise. Foi considerada para o estudo a maior medida. Os dados coletados foram registrados como quilograma força $(\mathrm{kg})$, e classificados conforme - Consenso Europeu de Sarcopenia de 2018, European Working Group on Sarcopenia in Older People (EWGSOP) no qual, para mulheres o ponto de corte é $<16 \mathrm{~kg}$ e para homens $<27 \mathrm{~kg}$, indicando baixa força muscular. Acima desses valores a classificação de força muscular é considerada preservada ${ }^{13}$.

\section{Teste de Desempenho Físico}

O teste de desempenho físico consistiu em caminhada por 4 metros, cronometrada, e após calculada a velocidade através da fórmula distância dividida pelo tempo $(\mathrm{m} / \mathrm{s})$. $O$ ponto de corte considerado foi de $\leq 0,8 \mathrm{~m} / \mathrm{s}$, sendo que 
resultados iguais ou abaixo indicam baixo desempenho físico ${ }^{8,14}$. O teste foi realizado após a sessão de HD.

\section{Critérios para Diagnóstico de Sarcopenia}

Foram classificados indivíduos com provável sarcopenia, aqueles que apresentaram apenas baixa FPM pela dinamometria. Foram classificados com sarcopenia, os pacientes que apresentaram redução da FPM, associada com redução da reserva muscular pela CP. Já o diagnóstico de sarcopenia grave deu-se aos indivíduos que apresentaram redução da FPM, associada com redução da massa muscular e redução do desempenho físico ${ }^{8}$.

\section{Análise Estatística}

Para análise estatística utilizou-se teste de Qui-Quadrado para associação de variáveis categóricas, gerando prevalências para o desfecho e $\mathrm{p}$-valor. $\bigcirc$ teste foi realizado no programa SPSS Stastistic Data 23.0. A distribuição amostral normal foi verificada com a aplicação do teste de ShapiroWilk, sendo as variáveis de distribuição categóricas e não paramétricas. Considerou-se um nível de significância de $5 \%(p \leq 0,05)$ para identificar associação entre as variáveis e o desfecho.

O estudo foi aprovado pelo Comitê de Ética e Pesquisa do Hospital Nossa Senhora de Pompéia, parecer n 2.612.951, e pelo Comitê de Ética e Pesquisa da FSG Centro Universitário, parecer $n^{\circ} 2.533 .095$. Os dados foram coletados após assinatura do Termo de Esclarecimento Livre e Esclarecido.

\section{RESULTADOS}

O ambulatório atendia 115 pacientes no total, destes, 12 negaram-se a participar, 4 possuíam membros amputados e 4 eram cadeirantes. Foram avaliados 95 pacientes, destes $21 \%(n=20)$ apresentaram algum grau de sarcopenia, 36,8\% $(n=35)$, provável sarcopenia, $8,4 \%(n=8)$, sarcopenia e $12,6 \%(n=12)$, sarcopenia grave. A Tabela 1 refere-se à caracterização da amostra, $61,1 \%(n=58)$ do sexo masculino, $53,7 \%(n=51)$ com idade $<60$ anos, sendo a média de idade de 55,4 $\pm 15,1$ anos. $O$ tempo de diálise mais prevalente foi de 1 a 5 anos, representando $44,2 \%(n=42)$. A média do IMC foi 25,9 $\pm 5,1 \mathrm{~kg} / \mathrm{m}^{2}$, sendo 45,3\% ( $\left.\mathrm{n}=43\right)$ com excesso de peso e $34,7 \%(n=33)$ apresentaram CP indicando depleção muscular.

A FPM indicou que $52,6 \%(n=50)$ dos indivíduos avaliados apresentavam fraqueza muscular, enquanto, sob o ponto de vista da performance muscular, $48,4 \%(n=46)$ apresentavam baixo desempenho físico.

A Tabela 2 refere-se à descrição das variáveis de exposição em relação à sarcopenia. Observou-se associação significativa entre idade e sarcopenia $(p \leq 0,0001)$, onde $60 \%$
Tabela 1 - Descrição das variáveis demográficas, tempo de hemodiálise, perfil nutricional e capacidade funcional em indivíduos com doença renal crônica em hemodiálise, em um hospital de Caxias do Sul/RS. 2019 ( $n=95)$.

\begin{tabular}{lc}
\hline Variáveis de exposição & $\mathbf{n}(\%)$ \\
\hline $\begin{array}{l}\text { Idade, em anos (x=55,4; } \mathbf{D P} \pm \mathbf{1 5 , 1 )} \\
\quad<60\end{array}$ & $51(53,7)$ \\
60 a 69 & $28(29,4)$ \\
70 a 79 & $11(11,6)$ \\
$\geq 80$ & $5(5,3)$ \\
\hline
\end{tabular}

\begin{tabular}{|c|c|}
\hline \multicolumn{2}{|l|}{ Sexo } \\
\hline Feminino & $37(38,9)$ \\
\hline Masculino & $58(61,1)$ \\
\hline \multicolumn{2}{|c|}{ Tempo de hemodiálise, em anos } \\
\hline$<1$ & $17(17,9)$ \\
\hline 1 a 5 & $42(44,2)$ \\
\hline$>5$ & $36(37,9)$ \\
\hline \multicolumn{2}{|c|}{ IMC (x=25,9 kg/m²; dp=5,1 kg/m²) } \\
\hline Baixo peso & $13(13,7)$ \\
\hline Eutrofia & $39(41,0)$ \\
\hline Excesso de peso & $43(45,3)$ \\
\hline \multicolumn{2}{|c|}{$\mathrm{CP}(\mathrm{x}=35,1 \mathrm{~cm} ; \mathrm{DP}=4,0 \mathrm{~cm})$} \\
\hline Adequado & $62(65,3)$ \\
\hline Depleção muscular & $33(34,7)$ \\
\hline
\end{tabular}

Força de Preensão Manual

Adequada força muscular

$45(47,4)$

Fraqueza muscular

$50(52,6)$

Teste de Marcha $(x=0,7 \mathrm{~m} / \mathrm{s} ; \mathrm{DP}=0,2 \mathrm{~m} / \mathrm{s})$

Adequado desempenho físico $49(51,6)$

Baixo desempenho físico $\quad 46(48,4)$

RS = Rio Grande do Sul; $\mathrm{x}$ = média; DP = Desvio padrão; $\mathrm{kg}$ = Quilograma; IMC = Índice de massa corporal; $\mathrm{CP}=$ Circunferência da Panturrilha; $\mathrm{kg} / \mathrm{m}^{2}$ = Quilograma por metro quadrado; $\mathrm{cm}=$ Centímetro; $\mathrm{m} / \mathrm{s}=$ Metros por segundo.

$(n=3)$ e $40 \%(n=2)$ daqueles com idade $\geq 80$ anos apresentavam sarcopenia e sarcopenia grave, respectivamente, e ainda, $60,8 \%(n=31)$ com idade $<60$ anos não tinham sarcopenia. Entre os pacientes com baixo peso segundo - IMC, 38,5\% ( $n=5)$ eram sarcopênicos graves e 30,8\% $(n=4)$ eram sarcopênicos. $46,5 \%(n=20)$ dos indivíduos com excesso de peso também foram diagnosticados com provável sarcopenia $(p \leq 0,0001)$. Em relação à $C P, 36,4 \%$ $(n=12)$ dos entrevistados com depleção de massa muscular apresentaram sarcopenia grave, já $51,6 \%(n=32)$ dos que tinham a $C P$ adequada demonstraram ausência de sarcopenia $(p<0,0001)$.

Ainda na Tabela 2, dentre os indivíduos com fraqueza muscular de acordo com a FPM, 24\% ( $n=12)$ apresentaram sarcopenia grave. Já, 88,9\% $(n=40)$ daqueles com força adequada não portavam sarcopenia. Segundo o teste de velocidade de marcha, $26,1 \%(n=12)$ dos que apresentaram baixo desempenho possuíam sarcopenia grave, e daqueles em que obtiveram desempenho adequado, $16,6 \%(n=8)$ eram sarcopênicos $(p<0,0001)$. 
Tabela 2 - Descrição das variáveis demográficas, tempo de hemodiálise, perfil nutricional e capacidade funcional em indivíduos com doença renal crônica em hemodiálise, em um hospital de Caxias do Sul/RS. 2019 (n=95).

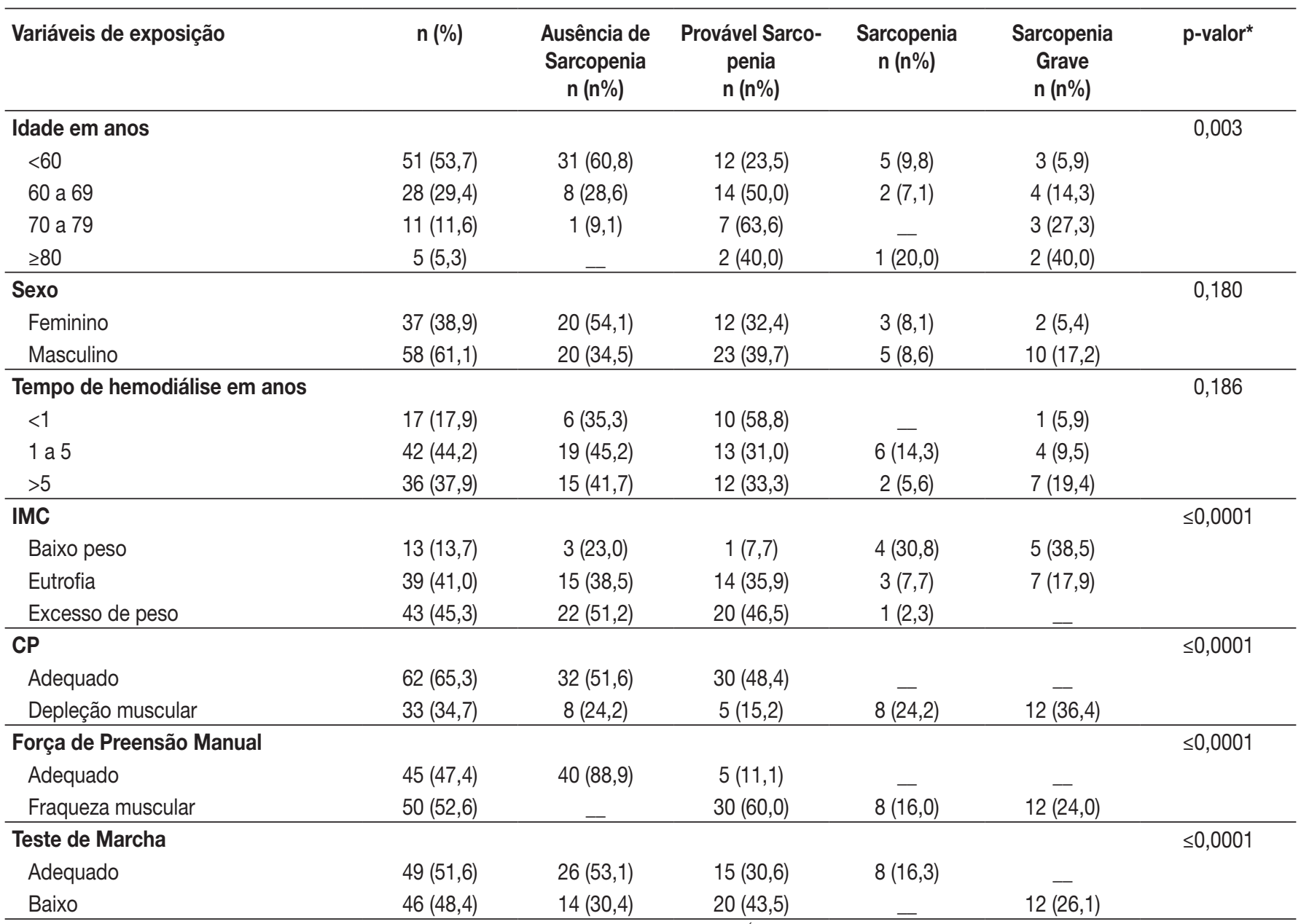

RS = Rio Grande do Sul; $n$ = Frequência bruta; $\mathrm{n} \%$ = Frequência relativa; $x=$ média; DP = Desvio padrão; IMC = Índice de massa corporal; CP = Circunferência da Panturrilha. *Teste Qui-Quadrado para heterogeneidade. Valores em negrito são estatisticamente significativos $(p \leq 0,05)$.

\section{DISCUSSÃO}

No presente estudo, o resultado mais significativo foi encontrado por meio da correlação entre IMC e presença de sarcopenia, ou seja, entre os indivíduos com baixo peso, a maioria foi significativamente correlacionada com a presença de sarcopenia. Embora muito utilizado, o IMC não é um instrumento preciso para obtenção de diagnóstico nutricional antropométrico, porém sabe-se que valores reduzidos de IMC estão relacionados com elevado risco de mortalidade ${ }^{15}$. A recomendação de IMC da OMS para indivíduos com idade $<60$ anos é a variação entre 18,5 e $24,9 \mathrm{~kg} / \mathrm{m}^{2}$, mas, o guia Europeu de nutrição para DRC recomenda $I M C>23 \mathrm{~kg} / \mathrm{m}^{2}$ para pacientes em $\mathrm{HD}$, visando um fator de proteção contra mortalidade precoce ${ }^{16}$. Os achados de um estudo publicado em 2016 por Johansen e Lee ${ }^{17}$ referem-se a maior mortalidade em pacientes que apresentaram valores de IMC baixos. Sabe-se que a reserva de massa muscular oferece efeito protetor para o avanço de doenças crônicas hipercatabólicas, como a DRC, entretanto, este estudo indica que a reserva adiposa também pode contribuir, a curto prazo, retardando a mortalidade dos pacientes acometidos pela DRC em HD ${ }^{17}$. Pacientes com baixo IMC e diagnosticados com sarcopenia pela baixa força e desempenho físico possuem duas vezes mais chances de óbito ${ }^{17}$. O desenvolvimento de sarcopenia ocorre pela redução de massa muscular e fatores associados, e não se correlaciona com o aumento e adiposidade, sendo esta a razão pela qual o IMC não é um parâmetro sensível para a condição sarcopênica ${ }^{18}$.

Nesta pesquisa, observou-se que $40 \%$ dos pacientes com idade $\geq 80$ anos apresentaram sarcopenia grave, e $20 \%$ tinham sarcopenia, isso ocorre principalmente pela própria condição do envelhecimento. Estudos indicam que idosos tendem a perder massa e função muscular ${ }^{19}$. Até 2010, - Consenso de Sarcopenia definia como uma condição exclusiva do idoso, entretanto, em 2018, houve uma revisão que associa a presença de sarcopenia com outros fatores, em especial com a presença de doenças de base, como no 
presente caso, insuficiência renal ${ }^{9}$. A incidência de sarcopenia em pacientes em HD em média é 13,7\%, aumentando gradualmente com a idade, chegando a $33,3 \%$ em pacientes com mais de 60 anos $^{20}$. Pacientes portadores de DRC em HD tendem a apresentar síndrome da fragilidade, em maior prevalência em idosos, além disso, são pacientes que apresentam tendência ao desenvolvimento do envelhecimento precoce, fato este que ocorre principalmente pelos sintomas da doença que incluem inapetência e a característica hipercatabólica da doença ${ }^{17}$.

Dentre as condições de risco para o desenvolvimento da sarcopenia, a baixa FPM esteve presente em $52,6 \%$ dos pacientes avaliados, sendo este um fator de risco agravante à mortalidade em doentes renais crônicos. Um estudo publicado em 2015 referiu que cerca de 50\% dos pacientes estudados apresentavam baixa FPM, ao que tudo indica, esse efeito pode ocorrer devido às complicações que o paciente sofre durante a sessão de hemodiálise, como hipotensão, fraqueza e fadiga ${ }^{21}$. No presente estudo, a FPM foi coletada durante a sessão de diálise, porém sempre ao início, visando tornar o dado mais fidedigno.

Outro aspecto analisado foi a depleção de massa muscular, avaliada através da CP, com a qual, 34,7\% dos indivíduos avaliados foram classificados com baixa reserva muscular. A medida da CP indica alterações na massa magra, que ocorrem naturalmente com a idade e com a redução de atividade física ${ }^{12}$. A perda de massa muscular está diretamente relacionada com baixos níveis de FPM, além de contribuir para o surgimento de desfechos desfavoráveis ${ }^{18}$. Os pacientes submetidos a HD tendem a apresentar reserva muscular reduzida, sendo os achados superiores a $25 \%$ dos pacientes, em ambos os sexos, com sinais de desnutrição ${ }^{22}$. Em pacientes renais em terapia substitutiva, isso ocorre com elevada frequência, devido a dietas com baixo teor calórico e proteico, pela uremia, que é responsável pela presença de anorexia, estas circunstâncias geram falha na produção de proteínas plasmáticas e síntese de albumina, provocando posteriormente desnutrição nestes pacientes $^{23}$. $O$ tratamento hemodialítico apresenta elevada eficiência, entretanto, os pacientes podem apresentar edema periférico, diante de tal situação é recomendado que aferições antropométricas sejam realizadas após a sessão de HD, por esta razão, a CP foi coletada após a sessão de diálise, tornando a medida veraz ${ }^{6}$.

Embora seja mais evidente a presença de sarcopenia em indivíduos com baixo peso, no presente estudo, $46,5 \%$ dos pacientes que apresentaram excesso de peso tinham provável sarcopenia. Tal fato é caracterizado pela preservação ou aumento do tecido adiposo em associação com perda de massa e qualidade do tecido muscular, tal condição é denominada obesidade sarcopênica ${ }^{17}$.
A escassez de estudos e testes voltados para avaliação de sarcopenia em pacientes com DRC em terapia dialítica faz com que a avaliação desta condição se dê apenas pelos critérios de diagnóstico estabelecidos pelo EWGSOP. Um estudo publicado em 2019 por Bae ${ }^{24}$ indica a prevalência de sarcopenia em pacientes com DRC entre 14\% e 63\%, e que estes pacientes tendem ao maior risco de desenvolvimento de doenças, infecções oportunistas e morte ${ }^{24}$.

Diante do desfecho do estudo, seriam estratégias de intervenção relevantes para a população estudada, o acompanhamento clínico multiprofissional precoce e contínuo, sendo descrito na literatura a eficácia da realização de atividades físicas, a correção de possível acidose metabólica, ingesta adequada de nutrientes, além da utilização de fármacos ${ }^{25}$.

Por ser um estudo de delineamento transversal, existem limitações, entre elas a falta de aferições contínuas, não correlação com possíveis patologias associadas, o que limita o estabelecimento de causa-efeito entre a sarcopenia e seus fatores associados. Além disso, a falta de uma avaliação nutricional mais detalhada para correlacionar com a sarcopenia e também a realização dos testes de FPM durante a sessão, e VM logo após a sessão, podem ter sido afetados pela fadiga induzida pela diálise. Dentre os pontos fortes do estudo, na região são escassas as pesquisas que relacionam sarcopenia com este público, o que impossibilita criação de técnicas para abrandar o problema.

atual estudo reflete significância e concordância com outros estudos do mesmo segmento. A sarcopenia apresenta correlação com a DRC principalmente em $H D$, visto que a condição está associada com menor qualidade de vida, prejudicando as condições físicas e alimentares desses indivíduos $^{23}$. A sarcopenia era antes uma condição associada apenas ao envelhecimento, entretanto, com melhor entendimento da síndrome, a ocorrência precoce da mesma tem sido estudada e reconhecida, sendo atualmente estudada em pacientes portadores de doenças crônicas hipercatabólicas ${ }^{8}$. Se faz necessário sequenciar estudos nesta perspectiva, visto que a condição sarcopênica nesses doentes está diretamente relacionada com o risco de complicações e maior mortalidade ${ }^{22}$.

\section{CONCLUSÃo}

As condições de provável sarcopenia e sarcopenia estiveram presentes na maioria dos pacientes estudados, especialmente nos doentes mais velhos e com baixo peso. Por meio deste estudo pode-se incentivar a criação de estratégias para melhorar a capacidade funcional e consequente qualidade de vida dos doentes renais crônicos em HD. Estudos ainda são necessários nesta perspectiva, visando a melhor avaliação das condições e da prevalência de sarcopenia em pacientes submetidos ao tratamento de HD. 


\section{REFERÊNCIAS}

1. Sesso RC, Lopes AA, Thomé FS, Lugon JR, Martins CT. Inquérito brasileiro de diálise crônica 2016. J Bras Nefrol. 2017;39(3):261-6.

2. Neves PDMM, Sesso RCC, Thomé FS, Lugon JR, Nascimento MM. Censo brasileiro de diálise: análise de dados da década 2009-2018. Braz J Nephrol. 2020;42(2):191-200.

3. Passos CB, Gallon CW. Estado nutricional e o seu impacto na saúde dos pacientes submetidos à hemodiálise: revisão de literatura. Rev Bras Nutr Clín. 2015;30(1):81-90.

4. Laegreid IK, Aasarod K, Bye A, Leivestad T, Jordhoy M. The impact of nutritional status, physical function, comorbidity and early versus late start in dialysis on quality of life in older dialysis patients. Ren Fail. 2014;36(1):9-16.

5. Barreto FC, Stinghen AEM, Oliveira RB, Franco ATB, Moreno AN, Barreto DV, et al. Em busca de uma melhor compreensão da doença renal crônica: uma atualização em toxinas urêmicas. J Bras Nefrol. 2014;36(2):221-35.

6. Montenegro MR, Walter RM, Morimoto JM, Paternez ACAC. Correlação dos métodos de avaliação nutricional de pacientes submetidosàhemodiálise. Rev SaúdeePesqui. 2015;8(2):267-75.

7. Ishikawa S, Naito S, Iimori S, Takahashi D, Zeniya M, Sato H, et al. Loop diuretics are associated with greater risk of sarcopenia in patients with non-dialysis-dependent chronic kidney disease. PLoS One. 2018;13(2):e0192990.

8. Cruz-JentoftAJ,BahatG, Bauer J, BoirieY,BruyèreO, Cederholm $\mathrm{T}$, et al; Writing Group for the European Working Group on Sarcopenia in Older People 2 (EWGSOP2), and the Extended Group for EWGSOP2. Sarcopenia: revised European consensus on definition and diagnosis. Age Ageing. 2019;48(1):16-31.

9. World Health Organization. Physical status: the use and interpretation of anthropometry. Report of a WHO Expert Committee. World Health Organ Tech Rep Ser. 1995;854:1-452.

10. Lipschitz DA. Screening for nutritional status in the elderly. Prim Care. 1994;21(1):55-67.

11. Barbosa-Silva TG, Bielemann RM, Gonzalez MC, Menezes AM. Prevalence of sarcopenia among community-dwelling elderly of a medium-sized South American city: results of the COMO VAI? study. J Cachexia Sarcopenia Muscle. 2016;7(2):136-43.

12. Oliveira Neto L, Agrícola PMD, Andrade FLJP, Oliveira LP, Lima KC. Qual o impacto do consenso europeu no diagnóstico e prevalência de sarcopenia em idosos institucionalizados? Rev Bras Gerontol. 2017;20(6):755-64.
13. Cruz-Jentoft AJ, Baeyens JP, Bauer JM, Boirie Y, Cederholm T, Landi F, et al; European Working Group on Sarcopenia in Older People. Sarcopenia: European consensus on definition and diagnosis: Report of the European Working Group on Sarcopenia in Older People. Age Ageing. 2010;39(4):412-23.

14. Lardiés-Sánchez B, Sanz-París A, Pérez-Nogueras J, SerranoOliver A, Torres-Anoro ME, Cruz-Jentoft AJ. Influence of nutritional status in the diagnosis of sarcopenia in nursing home residents. Nutrition. 2017;41:51-7.

15. Silva AMD, Souto TCM, Freitas FF, Morais CN, Souza BS. Estado nutricional de pacientes renais crônicos submetidos a tratamento hemodialítico em um hospital de referência de Pernambuco. Nutr Clín Diet Hosp. 2017;37(3):58-65.

16. Fouque D, Kalantar-Zadeh K, Kopple J, Cano N, Chauveau P, Cuppari L, et al. A proposed nomenclature and diagnostic criteria for protein-energy wasting in acute and chronic kidney disease. Kidney Int. 2008;73(4):391-8.

17. Johansen KL, Lee C. Body composition in chronic kidney disease. Curr Opin Nephrol Hypertens. 2015;24(3):268-75.

18. Souza VA, Oliveira D, Barbosa SR, Corrêa JODA, Colugnati FAB, Mansur HN, et al. Sarcopenia in patients with chronic kidney disease not yet on dialysis: analysis of the prevalence and associated factors. PLoS One. 2017;12(4):e0176230.

19. Martinez BP, Camelier FWR, Camelier AA. Sarcopenia em idosos. Rev Pesqui. em Fisioter. 2014;4(1):62-70.

20. Ren H, Gong D, Jia F, Xu B, Liu Z. Sarcopenia in patients undergoing maintenance hemodialysis: incidence rate, risk factors and its effect on survival risk. Ren Fail. 2016;38(3):364-71.

21. Pinto AP, Ramos CI, Meireles MS, Kamimura MA, Cuppari L. Impacto da sessão de hemodiálise na força de preensão manual. J Bras Nefrol. 2015;37(4):451-7.

22. Vasconcelos PAP, Tavares HC, Freitas LFF, Santos DG, Silva LSV, Bessa MMM, et al. Fatores associados à desnutrição em pacientes renais crônicos. Rev E-Ciência. 2018;6(1):54-60.

23. Gracia-Iguacel C, González-Parra E, Mahillo I, Ortiz A. Criteria for classification of protein-energy wasting in dialysis patients: impact on prevalence. Br J Nutr. 2019;121(11):1271-8.

24. Bae EH. Is sarcopenia a real risk factor for mortality in patients undergoing hemodialysis? Korean J Intern Med. 2019;34(3):507-9.

25. Moorthi RN, Avin KG. Clinical relevance of sarcopenia in chronic kidney disease. Curr Opin Nephrol Hypertens. 2017;26(3):219-28.

Local de realização do estudo: FSG Centro Universitário, Caxias do Sul, RS, Brasil.

Conflito de interesse: Os autores declaram não haver. 